\title{
Efeitos da Pentoxifilina sobre a Viabilidade in Vitro dos Espermatozóides de Eqüinos, após o Resfriamento a $5^{\circ} \mathrm{C}$
}

\author{
Herbert de Moura Goulart1, 2, Antônio Emidio Dias Feliciano Silva ${ }^{3}$, Concepta McManus ${ }^{4}$, \\ Frederico Ozanam Papa ${ }^{5}$
}

RESUMO - O objetivo deste trabalho foi avaliar, in vitro, o efeito da adição de $1 \mathrm{mg}$ pentoxifilina por $\mathrm{mL}$ de amostras seminais diluídas em meio Kenney, e resfriadas a $5^{\circ} \mathrm{C}$, de garanhões normospérmicos, sobre os seguintes parâmetros: taxas de motilidade total e motilidade progressiva, velocidade espermática ao longo da trajetória real, índice retilíneo, taxa de linearidade, velocidade progressiva, taxa de viabilidade e taxa de integridade da membrana plasmática. Foram avaliados 20 ejaculados de quatro garanhões, com o auxílio do Hamilton Thorn Research (Animal Version 12.0 L, EUA), em câmara de Makler. A taxa de viabilidade foi realizada por coloração de eosina e microscopia de contraste e a taxa de integridade de membrana, por coloração com fluorocromos, iodeto de propídio e diacetato de carboxifluoresceina e microscopia de fluorescência. As análises foram realizadas aos 30,60, e 120 minutos de incubação a $37^{\circ} \mathrm{C}$, após 12,24 e 48 horas de resfriamento a $5^{\circ} \mathrm{C}$. A pentoxifilina incrementou significativamente, em relação ao grupo controle, os parâmetros espermáticos relacionados à motilidade dos espermatozóides e de integridade da membrana plasmática, durante todo o período de incubação, da seguinte forma: taxa de motilidade total em $8,2 \%$; motilidade progressiva em 4,7\%; velocidade real em $23,1 \mathrm{~mm} / \mathrm{s}$; taxa de espermatozóides com velocidade rápida em 7,4\%; taxa de viabilidade em 4,7\%; e integridade da membrana plasmática em 3,5\% do tratado. Quanto aos parâmetros índice de retilinidade e linearidade, não foi observada diferença significativa entre os grupos. Os resultados obtidos nas condições deste experimento indicam que a adição de pentoxifilina ao ejaculado resfriado de garanhões, até 48 horas após o resfriamento, pode ser benéfica à qualidade dos espermatozóides para uso na inseminação artificial.

Palavras-chave: sêmen, garanhões, pentoxifilina, motilidade

\section{Effects of Pentoxifylline on the in vitro Viability of Equine Spermatozoids, after Cooling at $5^{\circ} \mathrm{C}$}

\begin{abstract}
The objective of this work was to evaluate the in vitro effect of the addition of $1 \mathrm{mg}$ pentoxifylline per $\mathrm{ml}$ of seminal samples from normospermic stallions, which were first diluted in Kenney extender and cooled at $5^{\circ} \mathrm{C}$. The evaluation was carried out using the following parameters: total and progressive motility rate, track speed, straightness and linearity rate, progressive velocity, viability rate and plasmatic membrane integrity rate. For this experiment, 20 ejaculates from four stallions were examined using Hamilton Thorn Research (Animal Version 12.0 L, EUA) with a Makler camera. The viability rates were obtained using eosin dyes under a light microscope and membrane integrity was obtained by fluorochrom, propidium iodine and carboxyfluorescein diacetat dyes under a fluorescence microscope. The semen examination occurred at 30,60 and 120 minutes of incubation at $37^{\circ} \mathrm{C}$, after 12,24 and 48 hours of cooling at $5^{\circ} \mathrm{C}$. The pentoxifylline significantly increased the sperm parameter rates of motility and plasmatic membrane integrity for all incubation times, when compared with the control group. The rates were: $+8.2 \%$ total motility, $+4.7 \%$ progressive motility, +23.1 $\mathrm{mm} / \mathrm{s}$ track speed, $+7.4 \%$ progressive velocity, $+4.7 \%$ viability and $+3.5 \%$ plasma membrane integrity. The straightness and linearity rates were not significantly different between the control and treated groups. The experimental data confirm that pentoxifylline addition to stallion-cooled sperm, up to 48 hours after cooling, could preserve sperm quality for artificial insemination.
\end{abstract}

Key Words: sperm, stallions, pentoxifylline, motility

\section{Introdução}

$\mathrm{Na}$ eqüinocultura, freqüentemente, o garanhão e a fêmea em reprodução encontram-se em locais distantes entre si. Neste caso, o tempo torna-se um fator limitante para a inseminação artificial com sêmen diluído resfriado e transportado em equitainer ou em qualquer outro meio de transporte similar.

Esta limitação é devida às grandes distâncias geográficas e problemas relacionados ao transporte

\footnotetext{
1 Aluno de Mestrado, Faculdade de Agronomia e Medicina Veterinária, Universidade de Brasília, CEP: 70910-900.

2 Policia Militar do Distrito Federal, BR060, Área Especial № 1, Granja Modelo, Riacho Fundo, DF.

3 EMBRAPA Recursos Genéticos e Biotecnologia, PqEB, Final W5 Norte, Brasília DF.

4 Professora, FAV/UnB, Brasília, DF, CEP: 70910-900.

5 Laboratório de Reprodução Animal, UNESP, Botucatu, SP
} 
deste sêmen da central de reprodução ao haras, o que afeta diretamente a qualidade do sêmen, pois com certa freqüência o sêmen resfriado e transportado chega ao seu destino com baixa taxa de viabilidade.

Considerando todos estes fatores, buscam-se novos métodos para prolongar a viabilidade do sêmen eqüino resfriado, maximizando o uso de garanhões e diminuindo os custos de produção, uma vez que o uso de sêmen congelado ainda apresenta questões técnicas a serem resolvidas, como um crioprotetor eficiente, havendo, também, resistência por parte de algumas associações de raça.

Entre os novos métodos, a adição da pentoxifilina, uma metilxantina, que promove o aumento do AMPc, responsável pela motilidade, é considerada uma droga promissora como um aditivo capaz de aumentar a viabilidade do sêmen resfriado e transportado, de eqüinos.

A pentoxifilina está sendo empregada nos casos de infertilidade de fator masculino levando em consideração a sua capacidade de promover a vida fértil dos espermatozóides. A pentoxifilina, segundo Yovich, (1993), auxilia na fertilização do ovócito durante os procedimentos de fertilização in vitro, promovendo o aumento da motilidade dos espermatozóides.

Diante deste quadro, o objetivo deste trabalho foi investigar e testar a adição de um produto, a pentoxifilina, a fim de potencializar a motilidade e sobrevida dos espermatozóides resfriados, minimizando os efeitos deletérios relacionados ao tempo em que o sêmen permanece fora do trato reprodutivo masculino ou feminino.

\section{Material e Métodos}

Foram utilizados quatro garanhões, sendo colhidos cinco ejaculados de cada, totalizando 20 amostras. Os garanhões apresentavam idade entre 5 e 13 anos, sendo os mesmos mantidos sob o mesmo regime de estabulação e manejo alimentar. As colheitas foram realizadas com intervalo de 48 horas. Não foi necessário esgotar os garanhões, pois os mesmos já permaneciam nesta rotina de colheita. A colheita de sêmen foi feita através de vagina artificial, modelo Botucatu (Papa et al., 1987), com auxílio de égua em cio e manequim.

Após a colheita, o sêmen foi filtrado e analisado os seguintes parâmetros: volume, motilidade subjetiva, vigor, concentração, $\mathrm{pH}$, osmolaridade, taxa de viabilidade de espermatozóides (teste supravital) e análise computadorizada realizada no Hamilton Thorn Research (Animal Version 12,0 L, EUA) em câmara de Makler (HTMA), com ajuste dos parâmetros sugeridos por Ferreira (2000).

Após colheita foram feitas as seguintes testes funcionais: teste hiposmótico e teste de integridade da membrana plasmática por fluorescência, de acordo com a técnica descrita por (Harrison et al.,1990), modificada por Zuccari (1998), e leitura em microscópio de fluorescência equipado com módulo de epiiluminação com fonte de halogênio e luz ultravioleta e lâmpada de mercúrio (Leica, Germany).

O preparo das amostras a serem analisadas por fluorescência ocorreu de seguinte forma: foram pipetados $40 \mu \mathrm{L}$ da solução de trabalho em tubo tipo eppendorf, protegido da luz em caixa de isopor, ao qual foram adicionados $10 \mu \mathrm{L}$ de sêmen. A leitura foi realizada, no máximo, duas horas após. Foram contadas 100 células em preparação úmida com lâmina e lamínula, sendo consideradas duas categorias para interpretação: espermatozóides íntegros ou espermatozóides lesados.

Os parâmetros mínimos para inclusão do ejaculado no experimento foram:

Volume - $20 \mathrm{~mL}$; Taxa de motilidade progressiva, subjetiva - 70\%, Vigor (movimento progressivo individual) - 2; Concentração - $35 \times 10^{6}$ (sptz/mL); Teste hiposmótico - $70 \%$ de sptz com flagelo enrolado; $\mathrm{pH}$ - 7,0; Osmolaridade - $250 \mathrm{mosmol} / \mathrm{L}$; Taxa de motilidade total (HTMA) - 75\%; Porcentagem de sptz com velocidade progressiva - 20\%; Velocidade real - $150 \mathrm{~mm} / \mathrm{seg}$; Índice de retilinidade - 60\%; Taxa de Linearidade - 30\%; Porcentagem de sptz com velocidade rápida - 55\%; Taxa de viabilidade pela coloração supravital - 60\%; Taxa de integridade de membrana plasmática por fluorescência - $60 \%$.

A avaliação do movimento espermático pós-incubação a $37^{\circ} \mathrm{C}$ foi realizada no Hamilton Thorn Research (Animal-Version 12,0L, EUA). Foram colocados $10 \mu \mathrm{L}$ de cada amostra na câmara de Makler e consideradas as avaliações propostas por Ferreira et al. (1997). Estes parâmetros foram avaliados aos 30, 60 e 120 minutos após a incubação a $37^{\circ} \mathrm{C}$ nas amostras obtidas às 12,24 e 48 horas após o início do resfriamento.

As seguintes avaliações foram computadas para análise estatística: Motilidade total da amostra, expressa em porcentagem; Motilidade progressiva, em porcentagem; Velocidade espermática ao longo da trajetória real, em $\mu \mathrm{m} / \mathrm{s}$; Índice retilíneo, em porcentagem; Linearidade, em porcentagem; e Número de espermatozóides com velocidade rápida, expresso em porcentagem. 
A análise da integridade da membrana plasmática foi realizada somente aos 60 minutos após a incubação a $37^{\circ} \mathrm{C}$ em banho-maria nas amostras obtidas às 12, 24 e 48 horas após o início do resfriamento.

A dose de 3,6 mMol, equivalente a $1 \mathrm{mg}$ de pentoxifilina por $\mathrm{mL}$ de sêmen diluído, foi estabelecida, a partir dos trabalhos de Shen et al. (1991), McKinney et al. (1996) e Centola et al. (1995, 1999).

A solução de pentoxifilina (PTX) foi preparada de uma vez para ser utilizada durante todo o experimento. A solução foi mantida à temperatura ambiente. $\mathrm{O}$ preparo da solução ocorreu a partir da adição de $400 \mathrm{mg}$ de (PTX) a $20 \mathrm{~mL}$ de solução de cloreto de sódio a $0,9 \%$ (Halex Star, Brasil), pH 5,87,320 mosm $/ 1$. O $\mathrm{pH}$ do cloreto de sódio foi previamente corrigido pela adição de $3 \mu \mathrm{l}$ de bicarbonato de sódio a 10\% (JP Farmacêutica, Brasil). Desta forma, obteve-se uma solução com $20 \mathrm{mg} / \mathrm{mL}, \mathrm{pH}$ 7,01 e 328 mosm/L.

Após a colheita e filtragem, uma amostra composta de $10 \mathrm{~mL}$ de sêmen foi adicionada a $10 \mathrm{~mL}$ do diluente de Kenney e acondicionada em tubo de poliestireno de $50 \mathrm{~mL}$ (Corning, EUA), o qual foi imergido em água (contida em caixa de isopor, a $37^{\circ} \mathrm{C}$ ). Em seguida, esta caixa foi colocada em conservador para sêmen suíno contendo termômetro digital (Minitub, Brasil), sendo as amostras resfriadas e mantidas a $5^{\circ} \mathrm{C}$.

Doze, vinte e quatro, e quarenta e oito após o início do resfriamento, foram retiradas duas alíquotas de $2 \mathrm{~mL}$ de cada amostra. A uma das alíquotas foram adicionados $100 \mu \mathrm{L}$ de pentoxifilina (Pentoxifylline, Sigma, EUA), correspondentes a $2 \mathrm{mg}$ de pentoxifilina, sendo esta alíquota denominada amostra PTX. A outra alíquota representou o controle, uma vez que não houve adição de pentoxifilina. As duas alíquotas foram incubadas em banho-maria a $37^{\circ} \mathrm{C}$, sendo realizadas análises de sêmen nos tempos 30, 60 e 120 minutos após o início da incubação. Os tempos de resfriamento e aquecimento foram estabelecidos na tentativa de reproduzir as condições reais encontradas durante os procedimentos de inseminação em eqüinos.

Os parâmetros analisados das amostras controle e das submetidas ao tratamento (resfriamento e adição de pentoxifilina) foram relacionados com as características do movimento espermático e com a integridade da membrana plasmática dos espermatozóides.

Os parâmetros de motilidade total, velocidade ao longo da trajetória real, motilidade progressiva, índice retilíneo, linearidade, porcentagem de espermatozóides com velocidade rápida, fluorescência e taxa de viabilidade (teste supravital) foram analisados segundo um modelo com as seguintes fontes de variação: repetição (combinação de garanhão e coleta), tratamento (pentoxifilina e controle), tempo de resfriamento, a 12, 24 e 48 horas, e interação entre tratamento e tempo de resfriamento. Os parâmetros motilidade total, velocidade espermática ao longo da trajetória real, motilidade progressiva, índice retilíneo, linearidade e porcentagem de espermatozóides com velocidade rápida foram avaliados ao longo dos três tempos de aquecimento aos 30,60 e 120 minutos, Estas avaliações foram consideradas como medidas repetidas, observadas na mesma unidade experimental e incorporadas ao modelo como um vetor de variável resposta. O comportamento destas variáveis está demonstrado na Figura 1.

A forma como estas medidas repetidas se comportaram para cada uma das fontes de variação do modelo determinou os tipos de testes utilizados para analisar os dados referentes a cada um destes parâmetros.

A primeira hipótese de interesse, que diz respeito à igualdade do perfil de médias ao longo dos três tempos de aquecimento e dos efeitos constantes do modelo, é de que estas são paralelas ao longo do período de aquecimento. Quando esta hipótese for aceita, os testes de interesse referem-se à igualdade das médias dos três tempos de aquecimento (tomadas sobre todos os tratamentos) e à igualdade dos efeitos constantes do modelo (tratamento e tempo de resfriamento), na média dos três tempos de aquecimento.

$\mathrm{Se}$, no entanto, a hipótese de paralelismo for rejeitada, as simplificações assumidas pela seqüência de testes descritos no parágrafo anterior não serão adequadas para representar os dados experimentais daquele parâmetro. Neste contexto, deve-se proceder à analise de variância segundo o modelo inicialmente proposto para cada tempo de aquecimento, sendo utilizado o PROC GLM do SAS (1999). Somente a velocidade espermática ao longo da trajetória real não apresentou paralelismo pelo teste de Wilks $(\mathrm{P}>0,05)$. Esta característica foi analisada como parcela subsubdivida, enquanto as outras (motilidade total, linearidade, índice retilíneo, motilidade progressiva e porcentagem de espermatozóides com velocidade rápida) foram analisadas como medidas repetidas.

As múltiplas comparações e intervalos de confiança (IC) foram obtidas pelo teste de Tukey $5 \%$. No caso dos parâmetros com medidas repetidas, utilizou-se, inicialmente, a opção REPEATED para realizar os testes das hipóteses de paralelismo e decomposição dos efeitos ao longo dos tempos de aquecimento. 

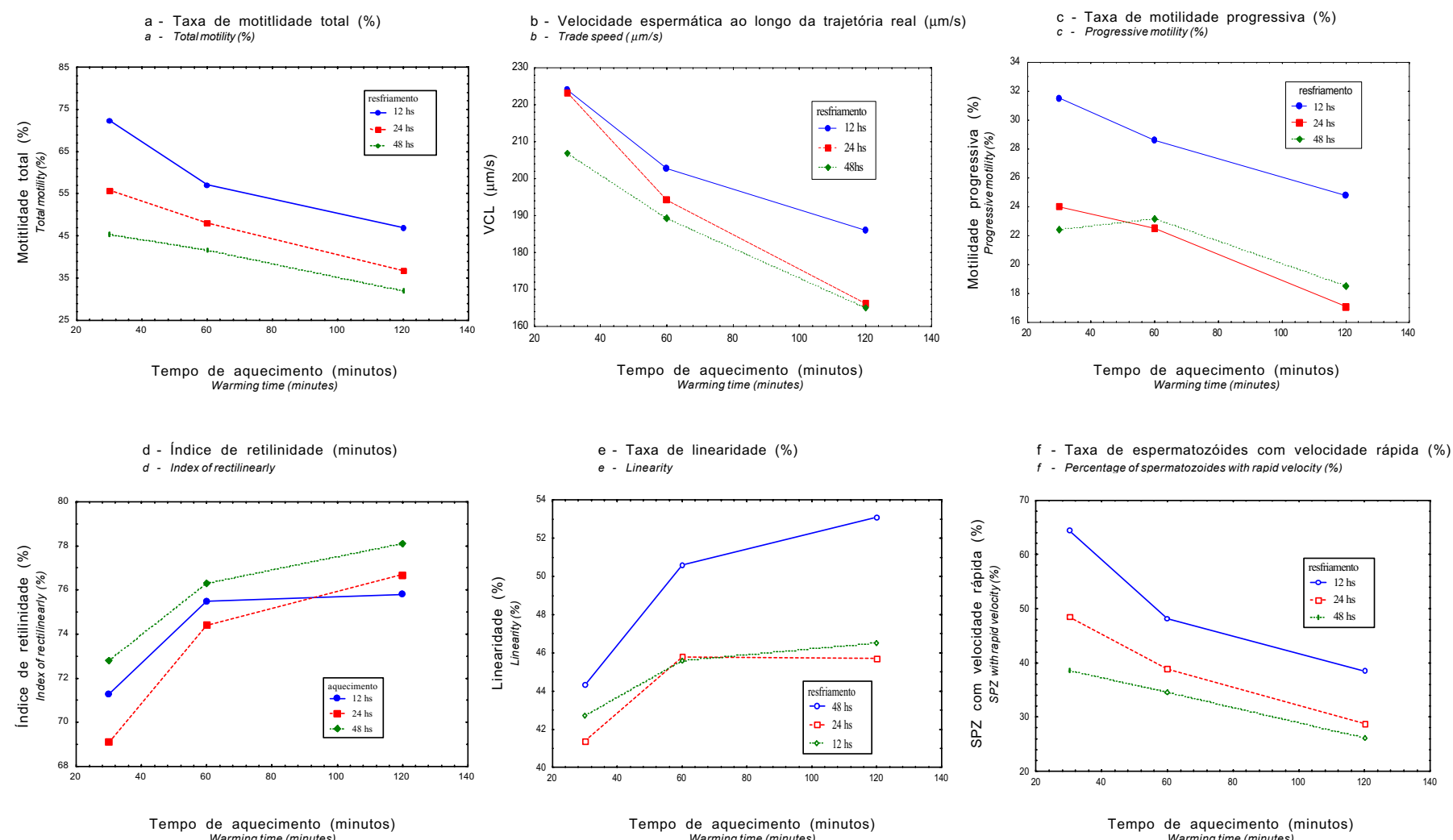

Figura 1 - Efeito de tempo de resfriamento e aquecimento sobre parâmetros em sêmen de garanhões. a - Taxa de motilidade total $(\%)$, b - Velocidade espermática ao longo da trajetória real $(\mu \mathrm{m} / \mathrm{s})$, c - Taxa de motilidade progressiva (\%), d - Índice de retilinidade (\%), e - Taxa de linearidade (\%), f - Taxa de espermatozóides com velocidade rápida (\%).

Figure 1 - Effect of cooling and warming on semen parameters in stallion: a - Total motility (\%), b - Trade speed ( $\mu$ m/s), c - Progressive motility (\%), $d$ - Index of rectilinearly (\%), e - Linearity (\%), $f$ - Percentage of spermatozoides with rapid velocity (\%).

\section{Resultados e Discussão}

O resumo dos dados do sêmen a fresco e resfriado, do grupo tratado com pentoxifilina e controle, está apresentado nas Tabelas 1 e 2 e o resumo da análise estatística de paralelismo, na Tabela 3.

Para todas as características examinadas, as médias das taxas dos três tempos de resfriamento não apresentaram comportamento paralelo (Tabela 3 ). O comportamento das características dentro dos tempos de aquecimento ao longo dos tempos de resfriamento foi, em geral, paralelo, exceto para motilidade total e porcentagem de espermatozóides com velocidade rápida. Os tratamentos mostraram o mesmo comportamento, exceto para a porcentagem de espermatozóides com velocidade rápida, que não foi paralela. A interação entre os três fatores (tempo de resfriamento, aquecimento e tratamento) foi paralela (Figura 1).

Os resumos das análises de variância para medidas repetidas e para tempo de resfriamento e aquecimento estão na Tabela 4 e por tratamento com tempo de aquecimento, na Tabela 5.

Para o índice de retilinidade e taxa de linearidade, não houve efeito significativo do tempo de resfriamento ou aquecimento (Tabela 4). O tempo de resfriamento afetou significativamente $(\mathrm{P}<0,05)$ os parâmetros porcentagem de espermatozóides com velocidade rápida, motilidade total, motilidade progressiva e porcentagem de espermatozóides com velocidade rápida, em todos os tempos de aquecimento exceto porcentagem de espermatozóides com velocidade rápida e motilidade progressiva aos 60 minutos. Em geral, os índices e taxas pioraram com tempo de resfriamento como esperado.

Quando se agruparam os tempos de resfriamento, o mesmo comportamento foi observado com o tratamento, índice retilíneo e linearidade não sendo afetado pelo tratamento independente de tempo de aque-

R. Bras. Zootec., v.33, n.1, p.112-122, 2004 


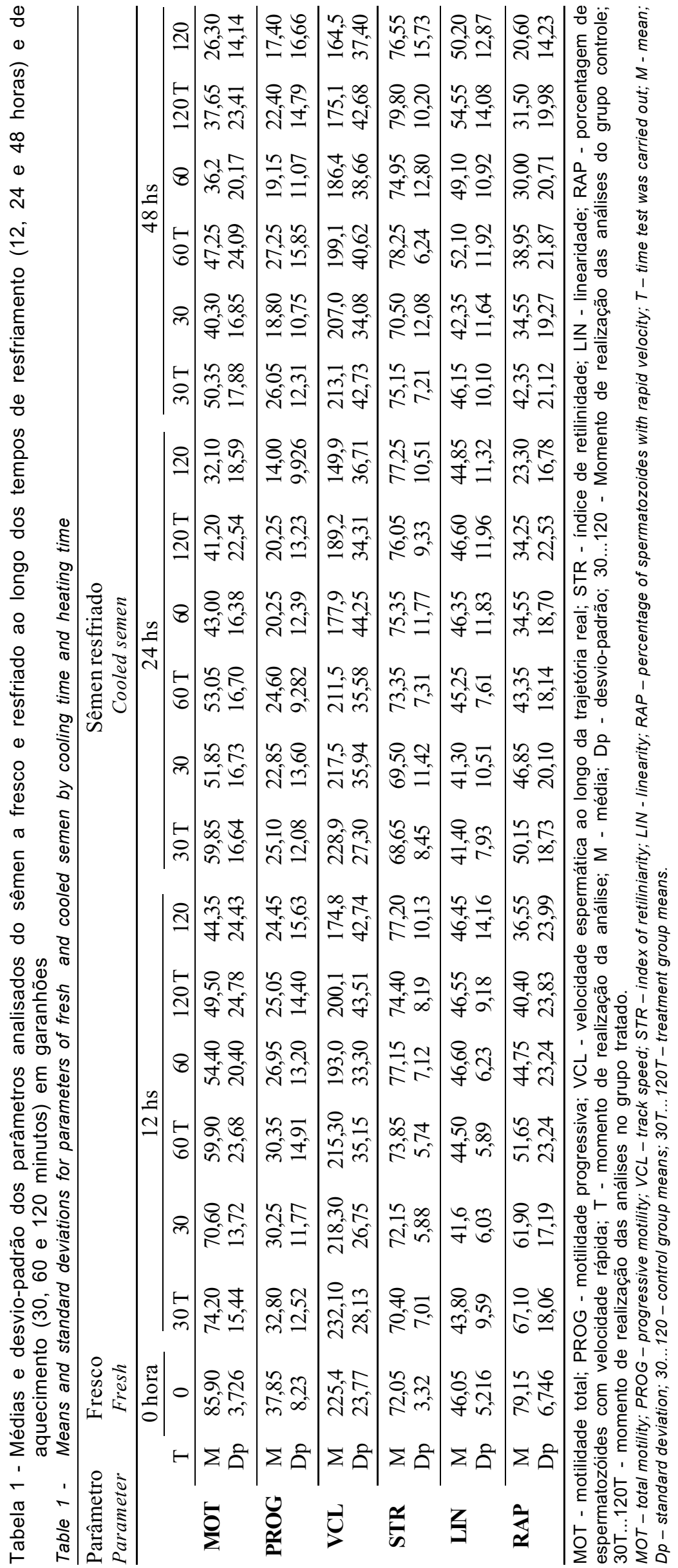


Tabela 2 - Médias e desvio-padrão das taxas de viabilidade e integridade da membrana plasmática de sêmen a fresco e resfriado, nos tempos de resfriamento 12, 24 e 48 horas e tempo de aquecimento de 60 minutos, em garanhões

Table 2 - Means and standard deviations for viability and plasma membrane integrity of fresh and cooled stallion semen with cooling times of 12, 24 and 48 hours and heating time 60 minutes

\begin{tabular}{|c|c|c|c|c|c|c|c|c|}
\hline \multirow[t]{3}{*}{$\begin{array}{l}\text { Parâmetros } \\
\text { Parameters }\end{array}$} & \multirow{3}{*}{\multicolumn{2}{|c|}{$\begin{array}{l}\text { A fresco } \\
\text { Fresh } \\
\text { M1 }\end{array}$}} & \multicolumn{6}{|c|}{$\begin{array}{l}\text { Resfriado } \\
\text { Cooled }\end{array}$} \\
\hline & & & \multicolumn{2}{|c|}{$12 \mathrm{~h}$} & \multicolumn{2}{|c|}{$24 \mathrm{~h}$} & \multicolumn{2}{|c|}{$48 \mathrm{~h}$} \\
\hline & & & PTX & CONT & M5T & M5 & M8T & M8 \\
\hline Viabilidade & $\mathrm{M}$ & 76,15 & 57,90 & 53,60 & 47,20 & 47,15 & 42,55 & 38,15 \\
\hline Viability & $\mathrm{Dp}$ & 8,20 & 14,90 & 16,27 & 12,31 & 12,95 & 14,05 & 13,24 \\
\hline Integridade & M & 75,80 & 63,15 & 58,00 & 48,10 & 45,10 & 39,90 & 37,50 \\
\hline Integrity & $\mathrm{Dp}$ & 8,35 & 12,95 & 13,65 & 10,33 & 11,60 & 11,76 & 14,02 \\
\hline
\end{tabular}

T - momento de realização da análise; M - média; Dp - desvio-padrão; M1...M8 - momento de realização das análises do grupo controle. M2T...M8T - momento de realização das análises no grupo tratado.

$T$ - analysis time; $M$ - mean; Dp - standard deviation; M1...M8 - control group tests; M2T...M8T - treatment group tests.

Tabela 3 - Valor de Wilks do teste de paralelismo e nível de significância para características de sêmen

Table 3 - Wilks value for parallelism test and significance level for semen traits

\begin{tabular}{lcccc}
\hline & $\begin{array}{c}\text { Tempo } \\
\text { aquecimento } \\
\text { Heating time }\end{array}$ & $\begin{array}{c}\text { Interação Tempo } \\
\text { Aquecimento } \\
\text { Tempo Resfriamento } \\
\text { Heating } x \text { Cooling time } \\
\text { interaction }\end{array}$ & $\begin{array}{c}\text { Interação Tempo } \\
\text { Aquecimento } \\
\text { Tratamento } \\
\text { Heating timex } \\
\text { Treatment interaction }\end{array}$ & $\begin{array}{c}\text { Interação Tempo } \\
\text { Aquecimento } x \text { Tratamento x } \\
\text { Tempo resfriamento } \\
\text { Heating } x \text { Cooling time } x \\
\text { Treatment interaction }\end{array}$ \\
\hline MOT & $0,3924^{* * *}$ & $0,8621^{* * *}$ & $0,9955^{\mathrm{ns}}$ & $0,9992^{\mathrm{ns}}$ \\
LIN & $0,6925^{* * *}$ & $0,9410^{\mathrm{ns}}$ & $0,9756^{\mathrm{ns}}$ & $0,9876^{\mathrm{ns}}$ \\
STR & $0,6279^{* * *}$ & $0,9716^{\mathrm{ns}}$ & $0,9924^{\mathrm{ns}}$ & $0,9981^{\mathrm{ns}}$ \\
PROG & $0,7627^{* * *}$ & $0,9711^{\mathrm{ns}}$ & $0,9954^{\mathrm{ns}}$ & $0,9866^{\mathrm{ns}}$ \\
RAP & $0,4175^{* * *}$ & $0,8798^{*}$ & $0,9896^{\mathrm{ns}}$ & $0,9838^{\mathrm{ns}}$ \\
VCL & $0,2781^{* * *}$ & $0,9059^{\mathrm{ns}}$ & $0,8708^{* * *}$ & $0,9611^{\mathrm{ns}}$
\end{tabular}

MOT - motilidade total; PROG - motilidade progressiva; VCL - Velocidade espermática ao longo da trajetória real; STR - índice de retilinidade; LIN - linearidade; RAP - porcentagem de espermatozóides com velocidade rápida; ${ }^{* \star *} \mathrm{P}<0,001 ;{ }^{*} \mathrm{P}<0,05$; ns - não significativa.

MOT - total motility; PROG - progressive motility; VCL - velocity of sperm along the true trajectory; STR - index of retiliniarity; LIN - linearity; RAP - percentage of spermatozoids with rapid velocity; ${ }^{* * *} P<.001 ;{ }^{*} P<.05 ;$ ns - not significant.

cimento enquanto as outras características foram afetadas, o tratamento com pentoxifilina significativamente melhorando as características do sêmen (Tabela 4).

O potencial de fecundação in vivo do espermatozóide é manifestado pela habilidade de percorrer o trato reprodutivo da fêmea, capacitar-se e ligar-se à zona pelúcida, sofrer a reação acrossômica, penetrar o ovócito e participar do desenvolvimento embrionário. Para isso, deve transportar todos os componentes bioquímicos na membrana plasmática, que, inter-relacionados com os fluidos do oviduto, exerce, com eficiência, a sua função.

No entanto, a capacidade fecundante, bem como a motilidade, pode ser prejudicada pelo estresse térmico, principalmente quando o sêmen é resfriado ou congelado, afetando a distribuição dos componen- tes lipoprotéicos e a permeabilidade da membrana (Parks, 1997; Graham, 1996). Por isso, a utilização do sêmen resfriado de eqüino na inseminação artificial está sujeita a distúrbios de funcionalidade que afetam a taxa de fertilidade.

Para minimizar os efeitos provocados pelo estresse térmico, neste experimento buscou-se um indutor funcional, a pentoxifilina, que, adicionado à concentração de 3,6 mM ao ejaculado diluído e resfriado a $5^{\circ} \mathrm{C}$ de eqüinos, in vitro, melhorou a maioria dos parâmetros espermáticos relacionados à motilidade. $\mathrm{O}$ efeito básico do incremento da motilidade nas amostras seminais tratadas com pentoxifilina ocorreu, provavelmente, por aumento da concentração intracelular de nucleotídeos cíclicos, em especial do AMPc, e da guanosina monofosfato cíclica (GMPc) como sugerido por Tesarik et al. (1992). Sabe-se que

R. Bras. Zootec., v.33, n.1, p.112-122, 2004 
Tabela 4 - Efeitos médios do tempo de resfriamento sobre as características do sêmen em tempos diferentes de aquecimento (tratamento e controle agrupados)

Table 4 - Mean cooling time effects on semen traits over different warming times (grouped over treatment and control)

Tempo de resfriamento (horas)

Tempo de aquecimento (minutos)

Warming time (minutes)

Cooling time (hours)

$30 \quad 60$

120

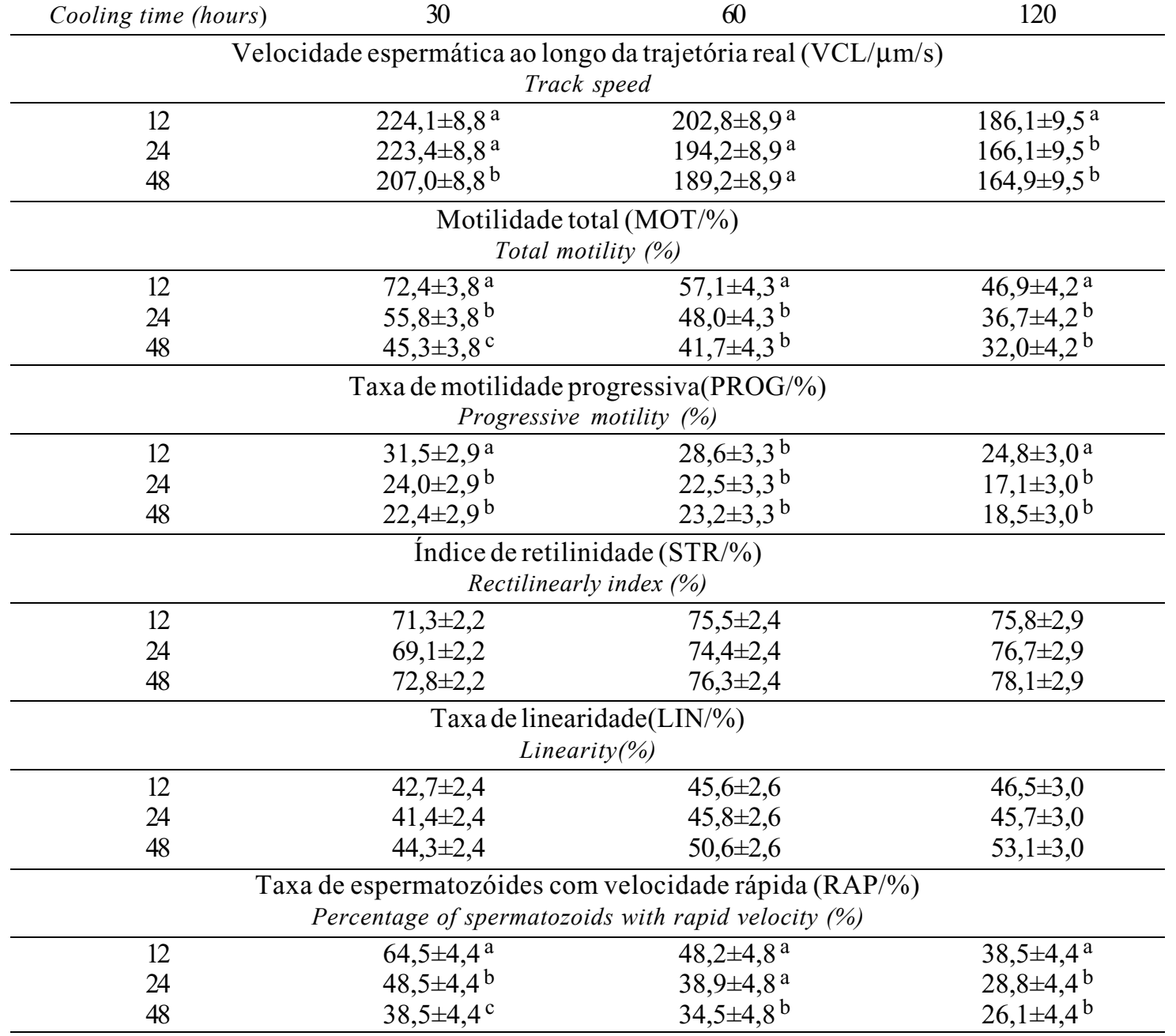

Médias em cada coluna e conjunto seguidas da mesma letra não diferem pelo teste Tukey $5 \%$.

Means in the same column and same trait with the same letter do not differ using Tukey test at 5\% significance level.

o AMPc interfere na indução da motilidade flagelar através da fosforilação protéica, liberando energia para dar início à atividade da dineina ATPase, responsável pela movimentação flagelar do espermatozóide (Perreault et al., 1982; Vijayraghavav et al., 1996). Ainda, para o aumento do AMPc, a pentoxifilina atua inibindo a fosfodiesterase e ativando a adenilciclase, que apresentam efeitos diretos no controle flagelar do espermatozóide (Manetta, 1998; Saberwal et al., 2002).

Neste experimento, os efeitos da pentoxifilina sobre os parâmetros espermáticos já se iniciaram após 30 minutos de incubação (Tabela 1), concordando com os resultados obtidos nos estudos de Fuse et al. (1993), Paul et al. (1995) e Esteves et al. (1998).

As maiores diferenças sobre a maioria dos parâmetros espermáticos obtidos entre o grupo submetido ao tratamento com pentoxifilina e o controle foram observadas aos 120 minutos de incubação (Tabela 5), fato também verificado por Manetta. (1997) e Fuse et al. (1993).

A motilidade total do grupo submetido ao tratamento, na média dos três tempos de resfriamento, ao longo dos tempos de aquecimento, foi superior ao grupo controle, na ordem de $8,2 \%$, valor inferior aos $37 \%$, encontrado por Esteves (2001), usando $5 \mathrm{mM}$ 
Tabela 5 - Efeitos médios do tratamento sobre as características do sêmen em tempos diferentes de aquecimento (os tempo de resfriamento foram agrupados)

Table 5 - Mean effects of treatment on semen traits over different warming times (grouped over cooling times)

\begin{tabular}{|c|c|c|c|}
\hline & \multicolumn{3}{|c|}{$\begin{array}{l}\text { Tempo de aquecimento (minutos) } \\
\text { Warming time (minutes) }\end{array}$} \\
\hline & 30 & 60 & 120 \\
\hline \multicolumn{4}{|c|}{$\begin{array}{l}\text { Velocidade espermática ao longo da trajetória real }(\mathrm{VCL} / \mu \mathrm{m} / \mathrm{s}) \\
\text { Track speed }\end{array}$} \\
\hline $\begin{array}{c}\text { Tratamento } \\
\text { Treatment } \\
\text { Controle } \\
\text { Control }\end{array}$ & $\begin{array}{l}223,8 \pm 7,2^{\mathrm{a}} \\
212,5 \pm 7,2^{\mathrm{b}}\end{array}$ & $\begin{array}{l}208,6 \pm 7,2^{a} \\
182,1 \pm 7,2^{b}\end{array}$ & $\begin{array}{l}188,1 \pm 7,7^{\mathrm{a}} \\
156,6 \pm 7,7^{\mathrm{b}}\end{array}$ \\
\hline \multicolumn{4}{|c|}{$\begin{array}{c}\text { Motilidade total (Mot/\%) } \\
\text { Total motility (\%) }\end{array}$} \\
\hline $\begin{array}{c}\text { Tratamento } \\
\text { Treatment } \\
\text { Controle } \\
\text { Control }\end{array}$ & $\begin{array}{l}61,5 \pm 3,1^{\mathrm{a}} \\
54,3 \pm 3,1^{\mathrm{b}}\end{array}$ & $\begin{array}{l}53,4 \pm 3,5^{\mathrm{a}} \\
44,5 \pm 3,5^{\mathrm{b}}\end{array}$ & $\begin{array}{l}42,7 \pm 3,4^{\mathrm{a}} \\
34,2 \pm 3,4^{\mathrm{b}}\end{array}$ \\
\hline \multicolumn{4}{|c|}{$\begin{array}{c}\text { Taxa de motilidade progressiva }(\operatorname{Prog} / \%) \\
\text { Porgressive motility (\%) }\end{array}$} \\
\hline $\begin{array}{c}\text { Tratamento } \\
\text { Treatment } \\
\text { Controle } \\
\text { Control }\end{array}$ & $\begin{array}{l}28,0 \pm 2,3^{a} \\
24,0 \pm 2,3^{b}\end{array}$ & $\begin{array}{l}27,4 \pm 2,7^{a} \\
22,1 \pm 2,7^{b}\end{array}$ & $\begin{array}{l}22,6 \pm 2,5^{a} \\
17,7 \pm 2,5^{b}\end{array}$ \\
\hline \multicolumn{4}{|c|}{$\begin{array}{c}\text { Índice de retilinidade }(\mathrm{STR} / \%) \\
\text { Rectilinearity index }(\%)\end{array}$} \\
\hline $\begin{array}{c}\text { Tratamento } \\
\text { Treatment } \\
\text { Controle } \\
\text { Control }\end{array}$ & $\begin{array}{l}71,4 \pm 1,8 \\
70,7 \pm 1,8\end{array}$ & $\begin{array}{l}75,1 \pm 2,0 \\
76,7 \pm 2,0\end{array}$ & $\begin{array}{l}76,7 \pm 2,4 \\
77,0 \pm 2,4\end{array}$ \\
\hline \multicolumn{4}{|c|}{$\begin{array}{l}\text { Taxa de linearidade (LIN/\%) } \\
\text { Linearity (\%) }\end{array}$} \\
\hline $\begin{array}{c}\text { Tratamento } \\
\text { Treatment } \\
\text { Controle } \\
\text { Control }\end{array}$ & $\begin{array}{l}41,8 \pm 2,0 \\
43,8 \pm 2,0\end{array}$ & $47,4 \pm 2,1$ & $47,2 \pm 2,5$ \\
\hline \multicolumn{4}{|c|}{$\begin{array}{c}\text { Taxa de espermatozóides com velocidade rápida (RAP/\%) } \\
\text { Percentage of spermatozoids with rapid velocity (\%) }\end{array}$} \\
\hline $\begin{array}{c}\text { Tratamento } \\
\text { Treatment } \\
\text { Controle } \\
\text { Control }\end{array}$ & $47,8 \pm 3,6^{b}$ & $36,4 \pm 3,9^{b}$ & $26,8 \pm 3,6^{b}$ \\
\hline
\end{tabular}

Médias em cada coluna e conjunto seguidas da mesma letra não diferem pelo teste Tukey $5 \%$.

Means in the same column and same trait with the same letter do not differ using Tukey test at $5 \%$ significance level.

equivalente a $1,38 \mathrm{mg} / \mathrm{mL}$ de pentoxifilina no précongelamento em sêmen humano. Entretanto, Centola et al. (1995), utilizando pentoxifilina em doses superiores a $5 \mathrm{mg} / \mathrm{mL}$, em sêmen humano, constatou que esta dosagem foi prejudicial aos espermatozóides. Os citados autores trabalharam com indivíduos portadores de patologias relacionados à infertilidade, (oligospermia, astenozoospermia e oligoastenozoospermia).

Em todos os tempos de resfriamento e ao longo dos tempos de aquecimento, a motilidade total do grupo submetido ao tratamento apresentou-se superior à do grupo controle (Tabela 5), sendo que aos 30 minutos a diferença foi de 7,2\%; aos 60 minutos, de $8,9 \%$; e aos 120 minutos, de $8,5 \%$.

A maior diferença observada entre os grupos controle e tratamento, para o parâmetro motilidade total, foi no tempo de resfriamento de 48 horas e tempo de aquecimento de 120 minutos. Isto demonstra o efeito positivo da pentoxifilina, que, mesmo adicionada após 48 horas de resfriamento, ativou a 
motilidade até 120 minutos de observação. Este resultado pode indicar que a pentoxifilina atua também como fator antioxidante (Tesarik et al., 1992) e que espermatozóides com os componentes bioquímicos íntegros na superfície da membrana conservam, mesmo sob o estresse térmico, a atividade de motilidade. A pentoxifilina pode ter propiciado importante redução nos radicais livres de oxigênio, que são deletérios para a atividade celular. No entanto, Centola et al. (1995), utilizando dose de pentoxifilina de $20 \mathrm{mg} / \mathrm{mL}$, atingiram o nível tóxico, observando a motilidade zero em sêmen humano.

A motilidade progressiva, do grupo submetido ao tratamento, na média dos tempos de resfriamento ao longo dos tempos de aquecimento (Tabela 5) foi superior ao grupo controle em $4,7 \%$, percentual inferior ao observado por Esteves (2001), de 13\%.

Em todos os tempos de resfriamento e ao longo dos tempos de aquecimento, a pentoxifilina melhorou a motilidade progressiva, sendo que aos 30 minutos, a diferença foi de 4,0\%; aos 60 minutos, de 5,3\%; e aos 120 minutos, de 4,9\% do grupo controle.

A maior diferença observada entre os grupos foi no tempo de resfriamento de 24 horas e tempo de aquecimento de 120 minutos (Tabela 1). Provavelmente, o sêmen resfriado durante 24 horas já deve apresentar queda da motilidade progressiva, devido a fatores tóxicos, distúrbios na fluidez de membrana, baixo nível de AMPc e pH e aumento de fosfatases protéicas que bloqueiam o mecanismo de ativação flagelar (Jones, 1998).

Os resultados obtidos nesta pesquisa assemelhamse e aos de Gradil et al. (2000), que, avaliando a adição de 3,5 a 7,0 $\mathrm{mM}$ de pentoxifilina, em sêmen resfriado a $4^{\circ} \mathrm{C}$, observaram aumento de motilidade durante 48 horas, quando comparado ao controle. Os resultados foram ainda semelhantes aos de Azevedo et al. (1998), que, trabalhando com sêmen humano, concluíram que o emprego da pentoxifilina melhora a motilidade dos espermatozóides em grau e porcentagem, mas não aumenta a concentração de espermatozóides por mililitros, como observado por Schill et al. (1992).

Neste experimento, $100 \%$ dos indivíduos (garanhões) testados responderam ao tratamento com pentoxifilina; já na espécie humana, Esteves (2001), que utilizou a dose de $5 \mathrm{mM}$ no pré-congelamento e constatou que em $31 \%$ de indivíduos não houve resposta ao tratamento com pentoxifilina. Paul et al. (1995), que usaram $6 \mathrm{mM}$ no sêmen humano somente $10 \%$ dos indivíduos testados, apresentaram pequena resposta ou não responderam ao tratamento.
Os resultados desta pesquisa mostraram-se diferentes dos de Esteves (2001) e Paul et al. (1995), possivelmente, em função da dose por nós testada e, também, do tipo de indivíduos, que foram normospérmicos.

O índice retilíneo e a taxa de linearidade, fatores relacionados à motilidade, tanto no grupo controle como no tratado com pentoxifilina (Tabela 3 ), aumentaram progressivamente ao longo do tempo de aquecimento, devido, provavelmente, a algum fator bioquímico e estrutural, porém não foi observada diferença significativa $(p>0,05)$ entre os grupos tratado e controle.

A velocidade espermática ao longo da trajetória real (Tabela 5) do grupo tratado com pentoxifilina, apresentou diferença de magnitude crescente $(p<0,05)$ ao longo dos tempos de aquecimento, em relação ao controle. Estas observações foram semelhantes as de Lewis et al (1993), em sêmen humano.

A maior diferença observada entre os grupos controle e tratamento, para o parâmetro velocidade ao longo da trajetória real, foi no tempo de resfriamento de 24 horas e tempo de aquecimento de 120 minutos, indicando, provavelmente, que neste tempo de resfriamento e de aquecimento inicia-se o seu efeito deletério sobre os espermatozóides do grupo controle. Tabela 1. Às 12 horas de resfriamento, a funcionalidade das células ainda está em plena atividade, não havendo efeito destacado da presença da pentoxifilina como inibidor da fosfatase protéica. Porém, a partir desta fase, grande população de espermatozóides resfriada necessita de um indutor de componentes de atividade flagelar, pois a motilidade é dependente da atividade respiratória, do flagelo e da integridade da membrana.

Deve-se destacar que, como na motilidade progressiva, na velocidade do espermatozóide há um provável estresse oxidativo sob o efeito do resfriamento por longo período, que afeta o metabolismo celular, comprometendo os batimentos do flagelo (Jones, 1973; Tesarik, 1992). Observou-se, também, que a adição de pentoxifilina, que se conhece ter efeito de aumento de AMPc e, indiretamente, antitóxico, elevou a taxa de células móveis e velocidade para percorrer determinada trajetória, provavelmente em função do aumento do metabolismo celular do flagelo.

Considerando os tempos de resfriamento e ao longo do aquecimento, a porcentagem de espermatozóides com velocidade rápida do grupo submetido ao tratamento com pentoxifilina apresentou-se superior ao controle, sendo que aos 30 minutos a diferença foi de $5,4 \%$; aos 60 minutos, de $8,2 \%$; e aos 120 minutos, de $8,5 \%$. 
A maior diferença observada entre os grupos controle e tratamento, para o parâmetro porcentagem de espermatozóides com velocidade rápida, foi no tempo de resfriamento de 48 horas e tempo de aquecimento de 120 minutos (Tabela 1). Segundo Patrizio et al. (2002), a pentoxifilina aumenta significativamente a força intrínseca de espermatozóides em sêmen humano, normospérmico e astenozoospérmico, permanecendo o efeito da droga até três horas, o que confirma o efeito, observado no resfriamento de 48 horas e aquecimento de 120 minutos.

O sêmen eqüino é muito sensível ao estresse de temperatura (Pickett et al., 1993). A membrana plasmática é afetada pelo frio, causando alterações na permeabilidade, funcionais e metabólicas, afetando a motilidade e capacidade de fecundação. Foi observado que o efeito do frio é minimizado pela adição de pentoxifilina, fato, nesta pesquisa, verificado na taxa de viabilidade, tempo de vida, bem como na integridade da membrana plasmática, que permaneceram em níveis superiores ao grupo controle, quando avaliadas pela fluorescência. Estes resultados favoráveis também foram obtidos por Ponce et al. (1999), ao testarem pentoxifilina na concentração de $5 \mathrm{mM}$ em espermatozóides de rato.

A manutenção da membrana do espermatozóide íntegra é fundamental para a reação acrossômica, etapa que antecipa a penetração na zona pelúcida e fecundação (Parks, 1997).

A manutenção de maior taxa de membrana íntegra, com a adição da pentoxifilina, indica que, possivelmente, não houve alteração dos componentes lipoprotéicos, iônicos e enzimas durante o processo de reaquecimento, o que ocorre com mais freqüência quando não está adicionada (Tabela 6). Estes componentes atuam direta ou indiretamente na motilidade, capacitação, no prolongamento do tempo de vida e na habilidade de fecundação (Parks et al., 1981; Nauc \& Mujanath, 2000). Entretanto, para maior acuidade na determinação da capacidade funcional do sêmen tratado com pentoxifilina, serão necessários mais estudos que envolvam testes de inseminação e mesmo de fecundação in vitro (FIV).

Mesmo fazendo-se análises conjuntas de qualidade e quantidade de movimento, viabilidade, morfologia, estado do acrossomo e função espermática, podem-se identificar apenas as amostras que provavelmente não têm fertilidade. Porém, as amostras que mostram resultados satisfatórios em todos os testes podem não ter necessariamente sua fertilidade garantida (Zahn, 2002).

Para se comprovarem os efeitos benéficos da
Tabela 6 - Médias ( \pm desvio-padrão) da taxa de viabilidade (SV) e de integridade de membrana plasmática $(F)$, em função do tempo de resfriamento e tratamento em sêmen de garanhões

Table 6 - Means ( \pm standard deviation) of viability (SV) and plasmatic membrane integrity $(F)$ as a function of cooling time and treatment in stallion semen

\begin{tabular}{ccc}
\hline $\begin{array}{c}\text { Tempo de } \\
\text { resfriamento (horas) } \\
\begin{array}{c}\text { Cooling time } \\
\text { (hours) }\end{array}\end{array}$ & $\mathrm{SV}$ & \\
\hline 12 & $54,7 \pm 2,6^{\mathrm{a}}$ & $60,6 \pm 2,7^{\mathrm{a}}$ \\
24 & $46,2 \pm 2,6^{\mathrm{b}}$ & $46,6 \pm 2,7^{\mathrm{b}}$ \\
48 & $39,4 \pm 2,6^{\mathrm{c}}$ & $38,7 \pm 2,7^{\mathrm{c}}$ \\
& $49,1 \pm 2,1^{\mathrm{a}}$ & $50,4 \pm 2,2^{\mathrm{a}}$ \\
Tratamento & $44,4 \pm 2,1^{\mathrm{b}}$ & $46,9 \pm 2,2^{\mathrm{b}}$ \\
Controle &
\end{tabular}

Médias em cada coluna e conjunto seguidas da mesma letra não diferem entre si pelo teste Tukey $5 \%$.

Means in the same column and same trait with the same letter do not differ using Tukey test at $5 \%$ significance level.

adição da pentoxifilina ao sêmen resfriado, há necessidade de se realizarem testes de fertilidade.

\section{Conclusões}

A adição de 3,6 $\mathrm{mM}$ de pentoxifilina, equivalente a $1,0 \mathrm{mg} / \mathrm{mL}$, no ejaculado de eqüinos in vitro, diluído e resfriado a $5^{\circ} \mathrm{C}$, melhora a viabilidade espermática.

Os resultados indicam a necessidade de se realizarem outros estudos testando diferentes concentrações de pentoxifilina, seu uso na pré e póscongelação, avaliação do poder antioxidante, alteração no tempo de capacitação espermática e seus efeitos em ejaculados de indivíduos com baixas taxas de fertilidade.

\section{Agradecimento}

À Dra. Maria Marina Unanian, Dra. Célia Maria Torres Cordeiro e Dr. Carlos Frederico Martins, pela ajuda no desenvolvimento deste trabalho.

\section{Literatura Citada}

AZEVEDO, D.A.; CAMPANHA, J.F.; VIERA, M.A.F. et al. Avaliação da motilidade do espermatozóide estimulada pela pentoxifilina. Reprodução e Climatério, v.13, Supl.1, p.55-59, 1998

CENTOLA, G.M.; CARTIE, R.J.; COX, C. Differential responses of human sperm to varying concentrations of pentoxyfylline with demonstration of toxicity. Journal of Andrology, v.16, supl. 2, p.136-142, 1995. 
ESTEVES, S.C. Criopreservação de espermatozóides humanos previamente tratados com pentoxifilina: efeitos na motilidade, vitalidade e perfil acrossômico após o descongelamento. São Paulo: Escola Paulista de Medicina, 2001. 104p. Dissertação (Doutorado em Medicina) - Escola Paulista de Medicina, 2001.

ESTEVES, S.C.; SHARMA, R.K.; THOMAS Jr., A.J. et al. Treatment of human spermatozoa with pentoxifyline before freezing improves the post-thaw agonist-induced acrosome reaction rate. Human Reproduction, v.13, p.3384-3389, 1998.

FERREIRA, J.C.P.; NEVES NETO, J.R.; PAPA, F.O. Avaliação computadorizada das características espermáticas de garanhões com fertilidade comprovada. Revista Brasileira de Reprodução Animal, v.21, p. 131-132, 1997.

FERREIRA, J.C.P. Avaliação subjetiva e computadorizada do movimento espermático pós-descongelação do sêmen eqüino. Botucatu: Universidade Estadual Paulista, 2000. 93p. Tese (Doutorado em Medicina Veterinária) Universidade Estadual Paulista, 2000.

FUSE, H.; SAKAMOTO, M.; OHTA, S. et al. Effect of pentoxifylline on sperm motion. Archives of Andrology, v.31, p.9-15, 1993.

GRADIL, C.M.; BALL, B.A. The use of pentoxifylline to improve motility of cryopreseved equine spermatozoa. Theriogenology, v.54, p.1041-1047, 2000.

GRAHAM, J.K. Cryopreservation of stallion spermatozoa. Veterinary Clinic North American Equine Practice. v.12, p.131-147, 1996.

HARRISON, R.A.; VICKERS, S.E. Use of fluorescent probes to assess membrane integrity in mammalian spermatozoa.Journal of Reproduction and Fertility, v.88, p.343-352, 1990.

JONES, R.; MANN T. Lipid peroxidation in spermatozoa. Proceedings Royal Society London Research, v.184, p.103-107, 1973.

JONES, R. Spermiogenesis and sperm maturation in relation to development of fertilizing capacity. In: LAURIA, A.; GANDOLFI, F.; ENNE, G. et al. (Eds.) Gametes: development and function. Roma: Serono, 1998. p.205-218.

LEWIS, S.E.M.; MOOHAN, J.M.; THOMPSON, W. Effects of pentoxifylline on human sperm motility in normospermic individuals using computer-assisted analysis. Fertility and Sterility,v.59, p.418-423, 1993.

MACKINNEY, K.A.; LEWIS, S.E.; THOMPSON, W. The effects of pentoxifiline on the generation of reactive oxygen species and lipid peroxidation in human spermatozoa. Andrology, v.28, p.15-20,1996.

MANETTA, L.A. Efeito da pentoxifilina sobre a motilidade e concentração dos espermtozóides humanos de indivíduos inférteis portadores de oligozoospermia e astenozoospermia. Ribeirão Preto: Universidade de São Paulo, 1997. 59p. Dissertação (Mestrado em Medicina) Universidade de São Paulo, 1997.

NAUC, V.; MANJUNATH, P. Radioimmunoassays for bull seminal plasma proteins (BSP-A1/A2, BSP 30 -kilodaltons), and their quantification in seminal plasma and sperm. Biology of Reproduction, v.63, p.1058-1066, 2000.

PANG, S.C.; WILLIANS, D.B.; HANG, C. Effects of pentoxifylline on sperm motility and hyperactivated motility in vitro. Fertility and Sterility, v.59, p.465-467, 1993.

PAPA, F.O.; ALVARENGA, M.A. Inseminação artificial com sêmen a fresco. Revista Cavalo Mangalarga, v.12, p.16-23, 1987.

R. Bras. Zootec., v.33, n.1, p.112-122, 2004
PARKS, J.E. Hypothermia and mammalian gametes. In: KAROW, A.M.; CRITSER, J.K. (Eds.) Reproductive tissue banking. San Diego: Academic Press, 1997. p.229-261.

PARKS, J.E.; MEACHAM, T.N.; SAACKE, R.G. Cholesterol and phospholipid of bovine spermatozoa. II. Effect of liposomes prepared from egg phsphotidylcholine and cholesterol on sperm cholesterol, phospholipids, and viability at $4^{\circ} \mathrm{C}$ and $37^{\circ} \mathrm{C}$. Biology of Reproduction, v.24, p.399-404, 1981.

PATRIZIO, P.; LIU, Y.G.; SONEK, G.J. et al. Effect of pentoxifilline on the intrinsic swimming forces of human sperm assessed by optical tweezers. Journal of Andrology, v.21, n.5, p.753-756, 2000.

PAUL, M.; SUMPTER, J.P.; LINDSAY, K.S. Action of pentoxifylline directly on semen. Human Reproduction, v.10, p.354-359, 1995.

PERRAULT, S.D.; ROGERS, B.J. Capacitation pattern of human spermatozoa. Fertility and Sterility,v.38, p.258-260, 1982.

PICKETT, B.W. Seminal extenders and cooled semen. In: McKINNON, A.O.; VOSS, J.L. (Eds.) Equine reproduction. Philadelfia: Lea \& Febiger, 1993. p.746-754.

PONCE, A.A.; DE CUNEO, M.F.; RUIZ, R.D. et al. Influence of pentoxifylline on sperm membrane functional integrity. Archives of Andrology, v.43, supl 1, p.77-88, 1999.

SABERWAL, G.S.; SHARME, M.K.; BALASINOR, N. et al. Estrogen receptor, calcium mobilization and rat sperm motility. Molecular and Cellular Biochemistry, v. 237, p.11-20, 2002.

STATISTICAL ANALYSIS SYSTEM - SAS. SAS/STAT ${ }^{\circledR}$ User's Guide. Version 8, Cary: 1999.

SCHILL, W.B.; MISKA, W. Possible effects of the kallikreinkinin system on male reproductive functions. Andrologia, v.24, n.2, p.69-75, 1992. (Review)

SHEN, M.R.; CHIANG, P.H.; YANG, R.C. et al. Pentoxifylline stimulates human sperm motility both in vitro and after oral therapy. Britsh Journal of Pharmacology, v.31, p.711714, 1991.

TESARIK, J.; THÉBAULT, A.; TESTART, J. Effect of pentoxifylline on sperm movement characteristics in normozoospermic and asthenozoospermic specimens. Human Reproduction, v.7, p.1257-1263, 1992.

VIJAYARAGHAVAN, S.; STEPHENS, D.T.; TRAUTMAN, $\mathrm{K}$. et al. Sperm motility development in the epididymis is associated with decreased glycogen synthase Kinase-3 and protein phosphatase 1 activity. Biology of Reproduction, v.54, p.709-718, 1996.

YOVICH, J.L. Pentoxifylline: actions and applications in assisted reproduction. Human Reproduction, v.8, 1786-1791, 1993.

ZAHN, F.S. Efeito da incorporação de colesterol na membrana plasmática de espermatozóides sobre os parâmetros espermáticos e índice de fertilidade do sêmen congelado na espécie eqüina. Botucatu: Universidade Estadual Paulista, 2002. 106p. Dissertação (Mestrado em Medicina Veterinária) - Universidade Estadual Paulista, 2002.

ZÚCCARI, C.E.S.N. Efeito da criopreservação sobre a integridade estrutural da célula espermática eqüina. Botucatu: Universidade Estadual Paulista, 1998. 121p. Tese (Doutorado em Medicina Veterinária) - Universidade Estadual Paulista, 1998. 